\title{
Facteurs et marqueurs de virulence de souches Escherichia coli isolées de diarrhées chez des veaux âgés de 4 à 45 jours en Algérie
}

\author{
A. Mohamed Ou Said ${ }^{1}$, M. Contrepois ${ }^{2,}$, M. Der Vartanian ${ }^{2}$, J.P. Girardeau ${ }^{2}$
}

MOHAMED OU SAID (A.), CONTREPOIS (M.), DER VARTANIAN (M.), GIRARDEAU (J.P.). Facteurs et marqueurs de virulence de souches Escherichia coli isolées de diarrhées chez des veaux âgés de 4 à 45 jours en Algérie. Revue Élev. Méd. vét. Pays trop., 1994, 47 (2) : 169-175

L'étude a porté sur 492 souches Escherichia coli isolées de matières fécales de 44 veaux diarrhéiques et de 4 veaux sains dans sept wilayates d'Algérie (Tipaza, Ain Defla, Bejaïa, Borj Bou Arreridj, Bouira, Médéa et Alger). Les auteurs ont recherché les protéines de surface K99, CS31A, Vir, F17 (FY), 20K et certains facteurs ou marqueurs de virulence tels que la production de colicines et en particulier de la colicine $V$, du sidérophore aérobactine, de l' $\alpha$ hémolysine et de l'entérohémolysine, et la fréquence de certains sérogronnes 0. Enfin, on a évalué la résistance des souches Escherichia coli à 10 antibiotiques. Les résultats montrent que la majorité des veaux diarrhéiques sont colonisés par des Escherichia coli exprimant un ou plusieurs facteurs de virulence et que les souches qui produisent l'antigène CS31A sont le plus souvent résistantes à 4 ou 6 antibiotiques.

Mots clés : Bovin - Veau - Diarrhée - Escherichia coli - Pouvoir pathogène - Souche - Protéine - Antigène - Antibiotique - Sérologie Algérie.

\section{INTRODUCTION}

Différentes protéines de surface confèrent aux Escherichia coli entéropathogènes des propriétés d'attachement leur permettant d'adhérer aux entérocytes et de coloniser l'intestin (3). Cette propriété, associée à d'autres facteurs de virulence comme la production d'entérotoxines ou de cytotoxines explique, pour une large part, le pouvoir pathogène des différents pathotypes de Escherichia coli. Pour caractériser les souches de Escherichia coli isolées chez les veaux atteints de diarrhées en 1990-1992, on a recherché les facteurs d'attachement K99 (27), F41 (20), Vir (26), F17 (16), 20K (1) et CS31A (14) ainsi que d'autres non identifiés à ce jour. Pour compléter cette étude, d'autres facteurs ou marqueurs de virulence ont été étudiés : la production de colicines et en particulier de la colicine $\mathrm{V}$, de l'aérobactine, de l' $\alpha$ hémolysine et de l'entérohémolysine. Enfin, on a identifié quelques sérogroupes 0 et étudié la résistance des souches Escherichia coli à différents antibiotiques.

1. Ecole nationale vétérinaire d'Alger, Laboratoire de microbiologie, BP 161, El. Harrach, 16200, Algérie.

2. INRA, Laboratoire de microbiologie, Centre de recherche de Clermont-Ferrand-Theix, 63122 Saint-Genès-Champanelle, France.

Reçu le 26.4.1993, accepté le 5.4.1994.

\section{MATÉRIELS ET MÉTHODES}

\section{Souches bactériennes}

Lors de passages dans des élevages, un prélèvement de matières fécales a été effectué chez des veaux diarrhéiques et quelques veaux sains. Les veaux étaient âgés de 4 à 45 jours, la majorité ayant entre 20 et 30 jours. Après dissémination sur gélose Mac Conkey (milieu sélectif pour entérobactéries), 10 à 12 souches de Escherichia coli ont été purifiées et identifiées sur galerios API 20E (La Balme les Grottes, France).

L'étude a porté sur 492 souches $E$. coli isolées chez 48 veaux diarrhéiques dont 2 veaux nouveau-nés d'une vache d'importation en attente dans un lazaret, répartis dans différentes régions d'Algérie (Ain Defla, Bejaïa Médéa, Bordj Bou Arréridj, Tipaza, Bouira et Alger). Les colibacilles de la microflore fécale de 4 veaux sains ont également été étudiés.

\section{Recherche des antigènes de surface K99, CS31A, Vir, F17 (FY) et 20K}

Les bactéries cultivées en boîtes de Pétri à la surface d'un milieu Minca gélosé (13) sont recueillies dans $2 \mathrm{ml}$ d'eau physiologique. Après chauffage à $60^{\circ} \mathrm{C}$ (28) et centrifugation, le surnageant est soumis à une électrophorèse en gel de polycrylamide en présence de SDS (15). Les marqueurs de poids moléculaire sont ceux du kit de calibration (Pharmacia Low Molecular Weight). Après coloration à l'argent, les bandes protéiques qui apparaissent à $18,5 \mathrm{kDa}, 20 \mathrm{kDa}, 21 \mathrm{kDa}$ et $29 \mathrm{kDa}$ correspondent respectivement aux sous-unités des antigènes K99, Vir ou 20K, F17 et CS31A. Ce résultat est complété par l'identification immunologique de ces protéines à l'aide d'anticorps spécifiques selon la technique d'immunoempreinte sur filtre.

\section{Recherche de la production de colicines et de la colicine $V$}

La recherche des colicines est effectuée selon la technique de la double couche (12). Les bactéries sont cultivées en "spot" à la surface d'un milieu de Luria gélosé. Après $24 \mathrm{~h}$ de culture à $37^{\circ} \mathrm{C}$, les bactéries sont lysées par les vapeurs de chloroforme pendant $30 \mathrm{~min}$ à $37^{\circ} \mathrm{C}$. 
Après aération, on coule une nouvelle couche de gélose molle $(0,7$ p. 100 d'agar $)$, pré-ensemencée par une souche de E.coli $\mathrm{K} 12$ sensible à toutes les colicines. L'inhibition de la croissance de la souche sensible aux colicines se traduit par des zones claires autour du "spot" après $24 \mathrm{~h}$ d'incubation à $37^{\circ} \mathrm{C}$.

La recherche de la colicine $V$ est réalisée uniquement chez les souches colicinogènes. On utilise comme souche indicatrice un mutant spontané défectif pour le récepteur de la colicine $\mathrm{V}$. Les souches qui produisent la colicine $V$ sont celles qui n'inhibent pas la souche résistante. Certaines souches Col $\mathrm{V}^{+}$peuvent éventuellement produire d'autres colicines.

\section{Recherche de la production d'aérobactine}

La synthèse du sidérophore de type hydroxamate (aérobactine) est mise en évidence par la technique de CSAKY (7) modifiée par DER VARTANIAN (8). Après culture des bactéries dans un milieu M9 (Prolabo) pauvre en fer pendant 24 heures à $37^{\circ} \mathrm{C}$ puis centrifugation à 3000 tours pendant $10 \mathrm{~min}$, on recueille $200 \mu$ l du surnageant de culture auquel on ajoute $30 \mu \mathrm{l}$ de la préparation suivante : $121 \mathrm{mg}$ de Chrome Azurol SLC (Aldrich) dans $60 \mathrm{ml}$ d'eau bidistillée à laquelle on ajoute $0,5 \mathrm{ml}$ d'une solution à $20 \mu \mathrm{M}$ de $\mathrm{FeC} 13$ et $10 \mathrm{mM}$ d'HCl ; après agitation pendant $10 \mathrm{~min}$, on ajoute $146 \mathrm{ml}$ d'héxadécyltriméthylammoniumbromide (Aldrich) mélangés à chaud dans $40 \mathrm{ml}$ d'eau bidistillée. Un virage du vert à une coloration orange ou jaune traduit la présence du sidérophore de type hydroxamate.

\section{Recherche de l'hémolysine et de l'entérohémolysine}

La recherche de l'hémolysine et de l'entérohémolysine a été réalisée par la méthode d'observation de la zone d'hémolyse autour des colonies après croissance sur milieu gélosé Blood Agar base additionné de 5 p. 100 de sang de mouton. La recherche de l'entérohémolysine a été effectuée selon la méthode de POHL et al. (23).

\section{La recherche des sérogroupes 0}

La recherche des sérogroupes $0(01,02,04,06,07,08$, $015,018,075,078)$ a été effectuée selon la technique d'immuno-empreinte sur filtre à l'aide d'antisérums spécifiques (Difco). Les extraits obtenus après chauffage à $60^{\circ} \mathrm{C}$ pour l'étude des antigènes de surface sont déposés sur des filtres de nitrocellulose. Après incubation avec des anticorps de lapin anti-0, les immunoglobulines fixées par les antigènes 0 sont reconnues par un anticorps anti IgG de lapin marqué à la péroxydase. L'activité enzymatique est révélée en présence d' $\mathrm{H}_{2} \mathrm{O}_{2}$ et d' $\alpha$ chloronapthol.

\section{Recherche de l'antibiorésistance}

L'antibiogramme a été effectué à l'aide de la méthode des disques (Institut Pasteur de Paris) sur milieu de Mueller Hinton gélosé. La surface de la gélose est ensemencée en la couvrant d'une dilution au 1/300 d'une culture de $18 \mathrm{~h}$ sur bouillon nutritif (Difco). Après élimination de l'excès de liquide et séchage de la surface de la gélose, les disques sont déposés. La lecture a lieu après $24 \mathrm{~h}$ d'incubation à $37^{\circ} \mathrm{C}$. On a étudié la résistance aux dix antibiotiques suivants : ampicilline $(10 \mu \mathrm{g})$, kanamycine $(30 \mu \mathrm{g})$, oxytétracycline $(30 \mu \mathrm{g})$, colistine $(50 \mu \mathrm{g})$, gentamicine $(15 \mu \mathrm{g})$, acide nalidixique $(30 \mu \mathrm{g})$, chloramphénicol $(30 \mu \mathrm{g})$, triméthoprime $(5 \mu \mathrm{g})$, sulfamides $(200 \mu \mathrm{g})$, et streptomycine $(10 \mu \mathrm{g})$

\section{RÉSULTATS}

Le tableau I montre l'absence de souches E. coli K99 dans l'ensemble des élevages. La présence de souches de $E$. coli K99 a été mise en évidence uniquement chez un veau âgé de 4 jours né dans un lazaret d'une vache issue d'une importation récente. Des souches Escherichia coli produisant les antigènes CS31A, Vir, F17, 20K ont été identifićcs. En effet, 61 Escherichia coli portent

TABLEAU I Fréquence des antigènes de surface (pili) parmi les souches Escherichia coli isolées de diarrhées chez les veaux en Algérie.

\begin{tabular}{|c|c|c|c|c|c|}
\hline \multirow{3}{*}{ Régions } & \multicolumn{5}{|c|}{ Fimbriae } \\
\hline & $\begin{array}{l}\mathrm{F} 17 \\
(\mathrm{Fy})\end{array}$ & CS31A & $20 \mathrm{~K}$ & Vir & Autres $^{1}$ \\
\hline & $N(p .100)$ & $\mathbb{N}(p .100)$ & $\mathbb{N}($ p. 100) & $N($ p. 100$)$ & $N(p .100)$ \\
\hline $\begin{array}{l}\text { Ain Defla } \\
N=104\end{array}$ & \multirow{7}{*}{$9 \quad(19)$} & $2 \quad$ (2) & $1 \quad(1)$ & $10(10)$ & $16(15)$ \\
\hline $\begin{array}{l}\text { Médéa } \\
\qquad N=116\end{array}$ & & $45 \quad(39)$ & $11(10)$ & $13(11)$ & 19. (16) \\
\hline $\begin{array}{l}\text { Bejaïa } \\
\qquad N=40\end{array}$ & & & $8 \quad(20)$ & & \\
\hline $\begin{array}{c}\text { Bordj B.A. } \\
\quad N=47\end{array}$ & & & $25(53)$ & $7 \quad(15)$ & \\
\hline $\begin{array}{l}\text { Tipaza }{ }^{2} \\
\qquad N=133\end{array}$ & & & $13(10)$ & & 72 (54) \\
\hline $\begin{array}{l}\text { Bouira } \\
\qquad N=23\end{array}$ & & & & & 11 (48) \\
\hline $\begin{array}{l}\text { Alger } \\
\qquad N=29\end{array}$ & & $13 \quad(45)$ & $9 \quad(31)$ & $3(10)$ & $7 \quad(24)$ \\
\hline $\begin{array}{l}\text { TOTAL } \\
\qquad N=492\end{array}$ & 10 & 60 (12) & 68 (14) & $33 \quad(7)$ & $125(25)$ \\
\hline
\end{tabular}

$N:$ nombre de souches

pili non encore décrits de poids moléculaires apparents $18,19,19,5$ ou $21 \mathrm{KDa}$;

2 : en plus de ces 133 souches 21 souches Escherichia coli $\mathrm{K}^{2} 9^{+}$ont été isolées chez un veau mort de diarrhée à l'âge de 4 jours alors qu'll était en observation dans un lazaret de Chéraga (Tipaza). 
l'antigène CS31A (soit 12 p. 100) et 33 l'antigène Vir (soit 7 p. 100). Le pilus $20 \mathrm{~K}$ est présent chez les souches isolées dans toutes les wilayates sauf Bouira (soit $14 \mathrm{p}$. 100). Dix souches portent l'antigène F17 (soit 2 p. 100). Les souches CS31A sont présentes en majorité chez les veaux diarrhéiques des régions de Médéa, d'Alger et de Ain Delfa. Les souches F17 ont été isolées dans les wilayates de Bordj Bou Arreridj et de Tipaza. L'étude électrophorétique a aussi révélé la présence de bandes protéiques de poids moléculaires $18,19,5$ ou $21 \mathrm{kDa}$ (tabl. I, II) correspondant probablement à des pili d'attachement non encore décrits. Ces pili $(18,19,5$ ou 21 $\mathrm{kDa}$ ) sont présents parmi les souches Escherichia coli isolées dans plusieurs régions d'Algérie (25 p. 100) avec une fréquence élevée au niveau de la wilaya de Tipaza (54 p. 100).

Les productions d'aérobactine et de colicines, tout particulièrement de la colicine $V$, sont largement répandues parmi les souches de Escherichia coli isolées des veaux malades dans les wilayates de Médéa, Bejaia, Bordj Bou Arreridj et Tipaza (tabl. II). Le sérogroupe 07 est le plus fréquemment observé. Il est isolé dans toutes les wilayates. Le sérogroupe 015 est également assez fréquent. Le sérogroupe 08 est souvent associé aux souches de Escherichia coli CS31A ${ }^{+}$. L'étude des marqueurs de virulence (tabl. III) parmi les Escherichia coli produisant des pili d'attachement montre que les souches de Escherichia coli CS31A produisent souvent l'aérobactine (37 sur 61 soit 61 p. 100) et 5 sont colicinogènes. Celles produisant le pilus Vir produisent également l'aérobactine (16 sur 19, soit 84 p. 100). Enfin, parmi les souches $20 \mathrm{~K}$, peu produisent l'aérobactine. La production de l'hémolysine $\alpha$ et de l'entérohémolysine est peu fréquente. Elle concerne seulement 9 souches CS31A (15 p. 100), 4 souches Vir (21 p. 100), 3 souches $20 \mathrm{~K}$ ( 4 p.100) et 3 autres souches piliées ( 3 p. 100).

Les antibiogrammes mettent souvent en évidence des multirésistances. Les souches qui hébergent l'antigène CS31A sont le plus souvent résistantes à 4 ou 6 antibiotiques. Si l'on considère maintenant les résultats obtenus pour chaque veau (tabl. II), il apparaît que 1 ou plusieurs facteurs ou marqueurs de virulence ont été identifiés chez les colibacilles isolés de la plupart des veaux malades. Par opposition, chez les 4 veaux sains $\left(n^{\circ} 4,5\right.$, 6,7 , wilaya de Tipaza), les facteurs ou marqueurs de virulence étaient totalement absents chez les veaux 4,5 et 6 . Chez le veau 7 , seulement 2 des 10 souches $E$. coli étudiées produisaient la protéine $20 \mathrm{~K}$.

\section{DISCUSSION}

Les résultats de cette étude soulignent l'absence de colibacilles entérotoxinogènes $\mathrm{K} 9^{+}$dans les différents élévages. Cependant, des souches $E$. coli $\mathrm{K} 99^{+}$ont été isolées des matières fécales d'un veau né d'une vache issue d'une importation récente et en observation dans un lazaret. Ce veau est mort à l'âge de 4 jours en état de déshydratation. Ce résultat confirme les conclusions d'une étude antérieure (19) et celle de MARTEL et al. (17). En effet, les colibacilles entérotoxinogènes $\left({\left.\mathrm{K} 99^{+}\right)}^{+}\right.$ conduisant à la mort par déshydratation affectent surtout les veaux de moins de 5 jours. Il est encore prématuré de conclure définitivement à l'absence de colibacilles $\mathrm{K} 99^{+}$ dans d'autres élevages. En revanche, l'identification de souches $E$. coli $\mathrm{K} 9^{+}$à partir d'animaux importés appelle à une surveillance et à un suivi pour les diarrhées colibacillaires chez les veaux issus de génisses placées dans un lazaret à leur arrivée en Algérie.

Les protéines CS31A, Vir, F17 et 20K, identifiées dans cette étude, ont été décrites et associées à différentes pathologies colibacillaires du veau. Ainsi, CS31A est souvent produit par les colibacilles isolés de septicémies (4, 17) ou de gastro-entérites avec ataxie (11). L'antigène Vir décrit initialement par SMITH (27) provenait de souches de $E$. coli invasives produisant la toxine Vir. Cette dernière est maintenant décrite sous l'appellation CNF2 (cytonecrotizing factor) (10) parmi les souches $E$. coli isolées de diarrhées ou de septicémies bovines (21). Les souches E. coli ${\mathrm{F} 17^{+}}^{+}$(anciennement Fy) produisent aussi l'antigène K99 lorsqu'elles sont entérotoxiques (4). Ce n'est pas le cas dans cette étude et la virulence des souches $E$. coli $\mathrm{F}^{17^{+}}, \mathrm{K} 99^{-}$reste à préciser. Des souches de E. coli CS31 $\mathrm{A}^{+}$ou $\mathrm{F}_{17^{+}}$ont été isolées également au Japon (25). Ces auteurs ont montré qu'elles provoquent expérimentalement des septicémies mortelles chez les veaux dont l'intestin est colonisé par des colibacilles entérotoxinogènes. Enfin, les souches $E$. coli de référence ayant conduit à l'identification des fimbriae $20 \mathrm{~K}$ provenaient de veaux diarrhéiques (1). Ces fimbriae, qui confèrent des propriétés d'attachement aux entérocytes, sont des facteurs de colonisation de l'intestin du veau. II convient aussi de noter que les fimbriae $20 \mathrm{~K}$ sont souvent produites par les souches $E$. coli $\operatorname{CS}_{1} 1 \mathrm{~A}^{+}(6)$. Dans cette étude, 2 souches $E$. coli $20 \mathrm{~K}^{+}$ont été identifiées chez un veau sain, mais elles étaient sous-dominantes dans la flore colibacillaire fécale.

L'aérobactine qui permet à $E$. coli de capter le fer lié à la transferrine ou à la lactoferrine pour sa croissance in vivo, favorise également la colonisation de l'intestin (9), point de départ pour des septicémies après translocation de Escherichia coli hors de l'intestin. Les colicines ne sont pas des facteurs mais des marqueurs de virulence. Les gènes plasmidiques codant pour l'aérobactine sont souvent associés sur un même plasmide à ceux codant pour les colicines $(24,29)$. Enfin, si l'activité cytotoxique directe de l'hémolysine n'est pas clairement établie, cette dernière est toujours associée à la production de la cytotoxine CNF1 (23).

En prenant comme base les diverses données sur les facteurs et marqueurs de virulence, les résultats obtenus au cours de cette enquête montrent que la quasi-totalité des veaux diarrhéiques sont colonisés par des souches $E$. coli exprimant un ou plusieurs facteurs ou marqueurs de virulence. Les antigènes de surface CS31A, Vir, K99, 
A. Mohamed Ou Said M. Contrepois M. Der Vartanian J.P. Girardeau

TABLEAU II Facteurs et marqueurs de virulence et antibiorésistances identifiés parmi les souches de Escherichia coli isolées chez chacun des veaux, dans les différentes wilayates.

\begin{tabular}{|c|c|c|c|c|c|c|}
\hline Régions & $\begin{array}{c}\mathrm{N}^{\circ} \\
\text { des veaux }\end{array}$ & Fimbriae & $\begin{array}{l}\text { Aérobactine } \\
\text { Colicines }\end{array}$ & $\begin{array}{c}\text { Hémolysine } \alpha \\
\text { Entérohémolysine }\end{array}$ & Sérogroupes & Résistance aux antibiotiques \\
\hline $\begin{array}{l}\text { Borj Bou } \\
\text { Arreridj } \\
\text { Tipaza }\end{array}$ & $\begin{array}{r}1 \\
2 \\
3 \\
4 \\
5 \\
6 \\
6 \\
7 \\
8 \\
9 \\
9 \\
10 \\
1 \\
2 \\
2 \\
3 \\
4 \\
1 \\
2 \\
3 \\
3 \\
4 \\
5 \\
6 \\
6 \\
9 \\
7 \\
10 \\
11 \\
12 \\
1 \\
2 \\
9 \\
10 \\
\end{array}$ & $\begin{array}{l}\text { Vir/20 K } \\
20 \mathrm{~K} \\
\text { Vir } \\
\text { CS31A } \\
\text { CS31A } \\
20 \mathrm{~K} \\
\text { Vir } \\
19 \mathrm{kDa} \\
\text { Vir } \\
19 \mathrm{kDa} \\
\text { Vir } \\
18 \mathrm{KDa} \\
\mathrm{CS} 31 \mathrm{~A} \\
20 \mathrm{~K} \\
\mathrm{Vir} / 20 \mathrm{~K} \\
\text { Vir/20 K } \\
\mathrm{CS} 31 \mathrm{~A} \\
\mathrm{CS} 31 \mathrm{~A} \\
\text { Vir } \\
\mathrm{CS} 31 \mathrm{~A} \\
\text { Vir } \\
20 \mathrm{~K} \\
20 \mathrm{~K} \\
\mathrm{CS} 31 \mathrm{~A} \\
\mathrm{CS} 31 \mathrm{~A} \\
\mathrm{CS} 31 \mathrm{~A} \\
\mathrm{CS} 31 \mathrm{~A} \\
\mathrm{CS} 31 \mathrm{~A} \\
\mathrm{CS} 31 \mathrm{~A} \\
\mathrm{~K} 99 \\
20 \mathrm{~K} \\
18 \mathrm{kDa}\end{array}$ & $\begin{array}{l}\text { Col V } \\
\text { Col V } \\
\text { Col V } \\
\text { Aéro } \\
\text { Aéro } \\
\text { Aéro } \\
\text { Aéro } \\
\text { Col V } \\
\text { Col V }\end{array}$ & 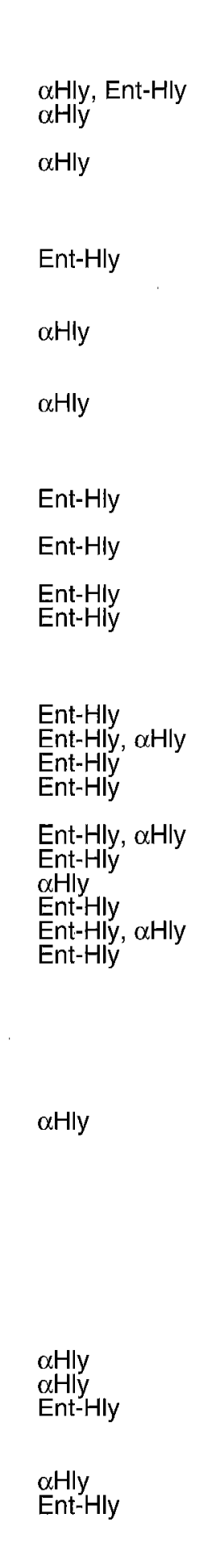 & $\begin{array}{l}015 \\
015 \\
\\
015 \\
07 \\
08 \\
015 \\
\\
02 \\
07 \\
07 \\
015 \\
07 \\
07 \\
\\
07 \\
\\
08 \\
07 \\
015 \\
\\
\\
078 \\
078 \\
\\
07 \\
015 \\
07 \\
07 \\
07 \\
07 \\
08 \\
07 \\
07 \\
08\end{array}$ & $\begin{array}{l}\text { Str, Suf, Tet } \\
\text { Tet } \\
\text { Tet, Amp } \\
\text { Kan, Str, Suf, Tet } \\
\text { Str, Suf, Tet, Amp } \\
\text { Kan, Str, Suf, Tet } \\
\text { Kan, Str, Suf } \\
\text { Tet } \\
\text { Tet } \\
\text { Tet } \\
\text { Tet } \\
\text { Cmp, Suf, Str, Tet, Amp } \\
\text { Kmp, Suf, Str, Tet, Amp, Tmp, Nalr } \\
\text { Str, Str, Suf, Tet, Amp } \\
\text { Set } \\
\text { Str, Tet }\end{array}$ \\
\hline
\end{tabular}




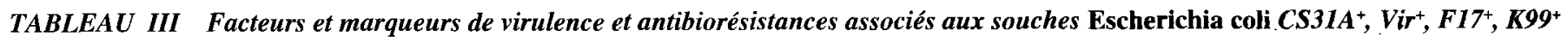
et $20 \mathrm{~K}^{+}$.

\begin{tabular}{|c|c|c|c|c|c|c|c|}
\hline & \multicolumn{2}{|c|}{$\begin{array}{l}\text { Colicines } \\
\text { et/ou Col V }\end{array}$} & \multicolumn{2}{|c|}{ Aérobactine } & \multicolumn{2}{|c|}{$\begin{array}{l}\text { Hémolysine } \alpha \text { et/ou } \\
\text { entérohémolysine }\end{array}$} & \multirow{2}{*}{$\begin{array}{l}\text { Résistance aux } \\
\text { antibiotiques }\end{array}$} \\
\hline & $N$ & (p. 100) & $\mathrm{N}$ & (p. 100) & $N$ & $(p .100)$ & \\
\hline $\begin{array}{l}\text { E. coli } \\
\text { CS } 31 \mathrm{~A} \\
\mathrm{~N}=61\end{array}$ & 5 & (8) & 37 & (61) & 9 & (15) & $\begin{array}{l}\text { Cmp, Kan, Str, } \\
\text { Suf, Tet, Amp }\end{array}$ \\
\hline $\begin{array}{l}\text { E. coli } \\
\text { Vir } \\
N=19\end{array}$ & 1 & (5) & 16 & (84) & 4 & (21) & Kan, Str, Suf, Tet \\
\hline $\begin{array}{l}\text { E. coli } \\
F 17 \\
N=10\end{array}$ & & & & & & & Tet \\
\hline $\begin{array}{l}\text { E. coli }{ }^{2} \\
\text { K99 } \\
\mathrm{N}=21\end{array}$ & & & & & & & $\begin{array}{l}\text { Kan, Str, Suf, Tet, } \\
\text { Amp }\end{array}$ \\
\hline $\begin{array}{l}\text { E. coli } \\
20 \mathrm{~K} \\
\mathrm{~N}=69\end{array}$ & 6 & (9) & 21 & (30) & 3 & (4) & $\begin{array}{l}\text { Kan, Str, Suf, Tet, } \\
\text { Amp }\end{array}$ \\
\hline $\begin{array}{l}\text { E. coli } \\
\text { avec } \\
\text { autres pili } \\
N=118\end{array}$ & 2 & (2) & 10 & (8) & 3 & (3) & $\begin{array}{l}\text { Kan, Str, Suf, Tet, } \\
\text { Amp }\end{array}$ \\
\hline
\end{tabular}

${ }^{1}$ Amp = ampicilline $; \mathrm{Kan}=$ kanamycine $;$ Tet = oxytétracycline $; \mathrm{Cmp}=$ chloramphénicol $;$ Tmp = triméthoprime $;$ Suf = sulfamides $;$ Str = streptomycine.

2 Souches Escherichia coli isolćcs chez un vcau mort de diarrhée à lâge de 4 jours alors qu'il était en observation dans un lazaret.

F17 et $20 \mathrm{~K}$ ont été identifiés parmi les souches $E$. coli provenant respectivement de 12,10 et 13 veaux diarrhéiques isolément ou parfois en association, soit au total chez 33 des 44 veaux diarrhéiques. La production de colicines ou de colicine $V$ est observée parmi les souches de $E$. coli isolées de 25 veaux diarrhéiques et celle de l'aérobactine à partir de 12 veaux diarrhéiques. L'hémolysine $\alpha$ ou l'entérohémolysine est produite par des souches de $E$. coli isolées de 18 veaux diarrhéiques. Par opposition à ces résultats, les souches de $E$. coli, isolées des matieres fécales de 4 veaux sains de la wilaya de Tipaza, ne possèdent pas ou peu les facteurs ou marqueurs de virulence. Seulement 2 des 10 souches $E$. coli chez le veau 7 produisaient les fimbriae 20K. Enfin, les résistances aux antibiotiques sont largement répandues.

Pour complèter cette étude, les travaux en cours visent à identifier les cytotoxines produites par les souches de $E$. coli. Celles produisant les fimbriae Vir synthétisent vraisemblablement la toxine CNF2 puisque les gènes Vir et CNF2 sont généralement associés sur un plasmide Vir (26). De même, parmi les souches hémolytiques, une partie produit vraisemblablement la toxine CNF1 puisque cette dernière est décrite à ce jour exclusivement en association avec la production d'hémolysine $(2,23)$. Enfin, les Vérotoxines de type Shiga like sont souvent décrites chez les souches $E$. coli isolées de l'intestin de veaux âgés de 1 jour à 3 mois et souffrant de diarrhées parfois associées à des troubles pulmonaires ou articulaires (22). Cette cytotoxine pourrait donc également expliquer la virulence de certains colibacilles isolés de veaux malades. Pour connaître de façon encore plus complète l'étiologie infectieuse des diarrhées des veaux, il faut également associer, à l'étude des colibacilles, celle des virus (rotavirus, coronavirus) et des parasites (cryptosporidies).

En conclusion, les résultats de cette étude indiquent que divers facteurs ou marqueurs de virulence peuvent expliquer une étiologie colibacillaire pour les diarrhées des veaux âgés de 4 à 45 jours. Il serait aussi utile de rechercher les souches $E$. coli $\mathrm{K} 99^{+}$chez les veaux diarrhéiques qui naissent dans les lazarets. L'étude en cours de réalisation pour identifier les cytotoxines, caractériser de nouvelles protéines impliquées dans l'attachement aux entérocytes et la recherche des virus et des parasites, devrait permettre de hiérarchiser l'importance des différents agents étiologiques responsables des diarrhées du veau en Algérie. L'identification des fimbriae impliquées dans l'attachement des colibacilles entéropathogènes est un préalable indispensable pour mettre au point une vaccination anti-attachement. Par ailleurs, cer- 


\section{A. Mohamed Ou Said M. Contrepois M. Der Vartanian J.P. Girardeau}

tains facteurs de virulence comme les toxines sont communs aux souches de $E$. coli pathogènes chez l'homme et l'animal. Le risque de zoonose étant possible, des retombées en santé humaine sont envisageables.

\section{REMERCIEMENTS}

Ce travail a fait l'objet d'un soutien financier des gouvernements algérien et français (Action DRS 92/73).

\section{BIBLIOGRAPHIE}

1. BERTIN (Y.), GIRARDEAU (J.P.). Etude d'unc nouvelle adhésine de type fimbriae chez les souches de $E$. coli d'origine bovine. 2e Congrès de la Société française de Microbiologie, Strasbourg, 18-21 sept. 1989, Abstract C9. p. 97 .

2. CAPRIOLI (A.), FALBO (V.), RUGGERI (F.M.), BALDASSARI (R.), BISSICHEA (G.), IPPOLITO (G.), ROMOLI (E.), DONNELLI (G.) Cytotoxic factor production by haemolytic strains of Escherichia coli causing extra intestinal infections. J. Clin. Microbiol., 1987, 25: 146-149.

3. CONTREPOIS (M.). Les colibacilles pathogènes : adhérence et facteurs de colonisation des colibacilles entérotoxinogènes. In : Symposium International "L'intestin grêle : physiologie, physiopathologie et pathologie". Evian (France), 1987. Excerpta med., 1987. p. 139-150.

4. CONTREPOIS (M.), MARTEL (J.L.), BORDAS (C.), HAYERS (F.) MILLET (A.), RAMISSE (J.), SENDRAL (R.). Fréquence des pili Fy et K99 parmi des souches de Escherichia coli isolées de veaux diarrhéiques en France. Annls Rech. vet., 1985, 16:25-28.

5. CONTREPOIS (M.), DUBOURGUIER (H.C.), PARODI (A.L.) GIRARDEAU (J.P.), OLLIER (J.L.). Septicaemic Escherichia coli and experimental infection of calves. Vet. Microbiol., 1986, 12: 109-118.

6. CONTREPOIS (M.), BERTIN (Y.), GIRARDEAU (J.P.), PICARD (B.) GOULLET (P.). Clonal relationship among bovine pathogenic Escherichia coli producing surface antigen CS31A. FEMS Microbiol. Letters, 1993, 108: $59-68$.

7. CSAKY (T.Z.). On the estimation of bound hydroxylamine in biological materials. Acta chem. scand., 1948, 2: 450-454.

8. DER VARTANIAN (M.). Differences in excretion and efficiency of the aerobactin and enterochelin siderophores in a bovine pathogenic strain of Escherichia coli. Infect. Immun., 1988, 56: 412-418.

9. DER VARTANIAN (M) JAFFEUX (B), CONTREPOIS (M), CHAVAROT (M.), GIRARDEAU (J.P.), BERTIN (Y.), MARTIN (C.). Role of aerobactin in systemic spread of an opportunistic strain of Escherichia coll from the intestinal tract of gnotobiotic lambs. Infect. Irnruur., 1992, 60: 2800-2807.

10. DE RYCKE (J.), GONZALEZ (E.A.), BLANCO (J.), OSWALD (E.), BLANCO (M.), BOIVIN (R.). Evidence of two types of cytotoxic necrotizing factors (CNF1 and CNF2) in human and animal clinical isolates of Escherichia coli. J. Clin. Microbiol, 1990, 28: 694-699.

11. ESPINASSE (J.), NAVETAT (H.), CONTREPOIS (M.), BAROUX (D.), SCHELCHER (F). $\Lambda$ new diarrhoeic syndrome with ataxia in young charolais calves: clinical and microbiological studies. Vet. Rec., 1991, 128: $422-425$.
12. FREDERICQ (P.). Colicines et colicinogenie. Annls Inst. Pasteur, $1964,107: 7-17$.

13. GUINEE (P.A.M), JANSEN (W.H), AGTERBERG (C.M.). Detection of the $\mathrm{K} 99$ antigen by means of agglutination and immuno electrophoresis in Escherichia coli isolated from calves and its correlation with enterotoxigenicity. Infect. Immun., 1976, 13: 1369-1377.

14. GIRARDEAU (J.P.), DER VARTANIAN (M.), OLLIER (J.L.), CON'TREPOIS (M.). CS3IA, a new K88 related timbrial antigen on bovine enterotoxigenic and septicaemic Escherichia coli strains. Infect. Immun., 1988, 56 2180-2188

15. LAEMMLI (U.K.). Cleavage of structural proteins during the assembly of the head of bacteriophage T4. London, Nature, 1990, 227: 680-685.

16. LINTERMANS (P.F.), POHL (P.), BERTELS (A.), CHARLIER (G.), VANDEKERCKHOVE (J.), VAN DAMME (J.), SCHOUP (J.), SCHLICKER (C.), KORHONEN (T.), DE GREVE (H.), VAN MONTAGU (M.). Characterization and purification of the F17 adhesin on the surface of bovine enteropathogenic and septicemic Escherichia coli. Am. J. vet. Res., 1988, 49: 1794-1799.

17. MARTEL (J.L.), CONTREPOIS (M.), DUBOURGUIER (H.C.), GIRARDEAU (J.P.), GOUET (PH.), BORDAS (C.), HAYERS (F.), QUILLERIET-ELIEZ (A.), RAMISSE (J.) SENDRAL (R.). Fréquence de l'antigène K99 et antibiorêsistance chez E. coli d'origine bovine en France. Annls Rech vét. 1981, 12: 109-263.

18. MOHAMED OU SAID (A.), CONTREPOIS (M.), DER VARTANIAN (M.), GIRARDEAU (J.P.). Virulence factors and markers in Escherichia coli from calves with bacteremia. Am. J. vet. Res., 1988, 49: 16571660 .

19. MOHAMED OU SAID (A.), CONTREPOIS (M.), DER VARTANIAN (M.), GIRARDEAU (J.P.), MOKRI (M.). Fréquence des facteurs et marqueurs de virulence et antibiorésistance chez les Escherichia coli isolés de diarrhées de veau chez le nouveau-né en Algérie. ler Congrès de la Fédération Méditérranéenne sur la Santé et la Production des Ruminants, Alghero, Italie, 2-3 mai 1991, Abstract p. 18.

20. MORRIS (J.), THORNS (C.), SCOTT (A.C.), SOJKA (W.J.), WELLS (G.). Adhesion in vitro and in vivo associated with an adhesive antigen (F41) produced by a K99 mutant of the reference strain Escherichia coli B41. Infect. Immun., 1982, 36: 1143-1146.

21. OSWALD (E.), DE RYCKE (J.), LINTERMANS (P.), VAN MUYLEM (K.), MAINIL (J.), DAUBE (G.), POHL (P.). Virulence factors associated wiht cytotoxic necrotizing factor type two in bovine diarrheic and septicemic strains of Escherichia coli. J. Clin. Microbiol., 1991, 29: 25222527.

22. Pohl (P.). Les Escherichia coli vërotoxinogènes isolées des bovins. Annls Med. vét., 1991, 135: 569-576.

23. POHL (P.), DAUBE (G.), MAINIL (J.), LINTERMANS (P.), KAECKENBEECK (A.), OSWALD (E.). Facteurs de virulence et phénotype de soixante et une souches d'Escherichia coli d'origines bovines productrices de la cytotoxine nécrosante de type 1 (CNF1). Annls Rech. vét., 1992, 23: $83-91$

24. SCIIOCII (C.), LEBEK (C.). Nachweiss von plasmidisch kodiertern eisen transport mechanismen in entero-bacteriaceae. Hyg. Med., 1984, 9 . 409-410.

25. SHIMIZU (M.), SAKANO (T.), YAMAMOTO (J.), KITAJMMA (K.). Incidence and some characteristics of fimbriae Fy and 31A of Escherichia coli isolates from calves with diarrhea in Japan. Microbiol. Immun., 1987, 31: $417-426$

26. SMITH (H.W.). A search for transmissible pathogenic characters in invasive strains of Escherichia coli: the discovery of a plasmid-controlled 


\section{BACTERIOLOGIE}

toxin and a plasmid-controlled lethal character closely associated, or identical, with colicine V. J. gen. Microbiol., 1974, 83: 95-111.

27. SMITH (H.W.), LINGGOOD (M.). Further observations on Escherichia coli enterotoxins with particular regard to those produced by atypical piglet strains and by calf and lamb strains: the transmissible nature of these enterotoxins and of a $\mathrm{K}$ antigen possessed by calf and lamb strains. J. med. Microbiol., 1972, 5: 243-250.

MOHAMED OU SAID (A.), CONTREPOIS (M.), DER VARTANIAN (M.), GIRARDEAU (J.P.). Factors and markers of virulence in Escherichia coli stocks isolated from diarrhoea in calves ages 4 to 45 days in Algeria. Revue Élev. Méd. vét. Pays trop., 1994, 47 (2) : 169-175

The study involved 492 strains of Escherichia coli isolated from the feces of 44 diarrhoeal calves and 4 healthy calves in 7 wilayates in Algeria (Tipaza, Ain Defla, Bejaïa, Borj Bou Arreridj, Bouira, Medea and Algiers). The authors lonked for the surface proteins K99, CS31A, Vir, F17 (FY), $20 \mathrm{~K}$ and certain factors or markers of virulence such as the production of colicins, particularly colicin $\mathrm{V}$, the aerobactin siderophore, $\alpha$ hemolysin and enterohemolysin. They also studied the frequency of certain 0 serogroups and evaluated the resistance of the $E$. coli strains to 10 antibiotics. The results showed that the majority of diarrhoeal calves were colonized by Escherichio coli expressing one or more factors of virulence and that the stocks which produce the antigen CS31A are usually resistant to 4 or 6 antibiotics.

Key words : Cattle - Calf - Diarrhoea - Escherichia coli - Pathogenicity Strain - Protein - Antigen - Antibiotics - Serology - Algeria.
28. STIRM (S.), ORSKOV (F.), MANSA (B.). Episome carried surface antigen K88 of $E$. coli II. Isolation and chemical analysis. J. Bact., 1967, 143: $731-739$

29. WILLIAMS (P.H.). Novel iron uptake system specified by col V plasmids: an important component in the virulence of invasive strains of Escherichia coli. Infect. Immun., 1979, 26 : 925-932.

MOHAMED OU SAID (A.), CONTREPOIS (M.), DER VARTA. NIAN (M.), GIRARDEAU (J.P.). Factores y marcadores de virulencia de cepas de Escherichia coli aisladas de diarreas de terneros de 4 a 45 días de edad, cn Argelia. Revue Élev. Méd. vét. Pays trop., 1994. 47 (2) : $169-175$

El estudio se llevó a cabo sobre 492 cepas de Escherichia coli aisladas de materias fecales de 44 terneros diarreícos y de 4 terneros sanos, en 7 "wilayates" de Argelia (Tipaza, Ain Defla, Bejaia, Borj Bou Arreridj, Bouira, Medea y Argel). Se buscaron las proteínas de superficie K99, CS31A, Vir, F17 (FY), 20K, así como ciertos factores o marcadores de virulencia, como la producción de colicinas, principalmente la colicina $V$, del sideróforn aerohactina, de la $\alpha$ hemolisina y de la enterohemolisina, así como la frecuencia de ciertos serogrupos 0. Finalmente, se calculó la resistencia de las cepas de $E$. coli ante 10 antibióticos. Los resultados demuestran una mayoría de terneros diarreícos colonizados por una Escherichia coli que presenta uno o más factores de virulencia y que las cepas que producen el antígeno CS31A son a menudo resistentes a 406 antibióticos.

Palabras clave : Bovino - Tenero - Diarrea - Escherichia coli - Poder patogeno - Cepa - Protéina - Antígeno - Antibiótico - Serología - Argelia. 